\title{
Failure Probability Analysis of Interlayer Sliding Belts of Gravity Dams Under Seismic Load
}

\author{
Qiang $\mathrm{Xu}^{*}{ }^{, 1}$, Jian-Yun Chen ${ }^{1,2}$, Chunfeng Zhao ${ }^{1}, \mathrm{Jing}_{\mathrm{Li}}{ }^{1}$ and Hongyuan Yue ${ }^{1}$ \\ ${ }^{1}$ School of Civil and Hydraulic Eng., Dalian University of Technology, Dalian, 116023, China \\ ${ }^{2}$ State Key Lab of Coastal and Offshore Eng., Dalian University of Technology, Dalian, 116023, China
}

\begin{abstract}
In this paper, an improved model is presented for analysis of failure probability of the interlayer sliding belts under seismic loads. Firstly, using the theory of the Markov chain, the relation between failure probabilities of specified interlayer sliding belts and elements in this interlayer sliding belt is deduced. Then, the failure function is proposed according to the destructive characteristic of concrete, the pseudo excitation method is utilized in order to obtain the probability distribution of element stresses in specified interlayer sliding belts, and the improved response surface method based on weighted regression is used to calculate the failure probability of elements in specified interlayer sliding belts. Finally, an algorithm is established to calculate the failure probability of the specified interlayer sliding belts. In this paper, the mean value and variance of the tensile strength of elements are changed when interlayer sliding belt is developed. The numerical results show that the conditional failure probability in specified interlayer sliding belts at the head of the dam tends to decrease. However, the tendency of conditional failure probability in the other specified interlayer sliding belts is complicated. And the interlayer sliding belt at head of the concrete gravity dam is the most dangerous. In addition, the tendencies of the mean value and mean square deviation of stresses in different specified interlayer sliding belts are similar. The mean value of stress in different specified interlayer sliding belts tends to decrease but the tendency of mean square deviation changes from decrease to increase. The range of the mean value and the mean square deviation of stress in specified interlayer sliding belts at the heel of the dam is the greatest of all.
\end{abstract}

Keywords: Gravity dam, seismic load, Markov chain, pseudo excitation method.

\section{INTRODUCTION}

It has now been widely recognized that the most reasonable method for dealing with multiple excitation problem is the random vibration approach. The pseudoexcitation method was utilized to solve the dynamic response of wind-excited structures with and without control devices [1]. It was also utilized to make random vibration analyses of structures under multi-component seismic excitations [2] and seismic responses of tall buildings [3]. With the method, [4] made probabilistic analysis for long-span structures such as long-span bridges. The method was further developed by [5] to improve the accuracy of dynamic response analysis of non-proportionally damped with multiple degrees of freedom discrete system. The sensitivity analysis formulae were derived for optimizing vehicle suspension systems based on the pseudo-excitation method [6], and a new algorithm was constructed based on the pseudo-excitation method to analyze the dynamic response of vehicles and to deduce the relationship between road excitation and vehicle responses [7]. The pseudo-excitation method, which is suitable for stochastic analysis of non-proportionally damped structure, was also improved by [8], and the method was also utilized

*Address correspondence to this author at the School of Civil and Hydraulic Eng., Dalian University of Technology, Dalian, China;

Tel: 0086-411-84709422; Fax: 0086-411-84709422;

E-mail: dlqiangxu@163.com to optimize the stiffness and damping parameters of suspension systems [9]. However, the pseudo-excitation method was only utilized to analyze linear structures because it was derived from superposition principle, which was only applied to linear structures.

In this paper, we propose the failure function according to the destructive character of concrete and the relation of failure probabilities between specified interlayer sliding belt and its elements based on the theory of Markov chain. Then we improve response surface method based on weighted regression from the aspects of the regression of sample points, the selection of experimental points, the determining method of weight matrix and the calculation method of point checking, respectively. Based on the pseudo excitation method, we demonstrate how to calculate the functional reliability, which is denoted as displacement of element $\mathrm{k}$ at dam head. Ultimately, a test example is utilized to verify and analyze the convergence and stability of the proposed method.

\section{MODEL FOR FAILURE PROBABILITY ANALYSIS IN SPECIFIED INTERLAYER SLIDING BELTS BASED ON THE MARKOV CHAIN}

\subsection{Failure Probability of Specified Interlayer Sliding Belts}

In this paper, the interlayer sliding belts were selected by searching the most probably sliding surface in the concrete 
gravity dam. It needs to be noticed that they are usually not the real interlayer sliding belts.

It is supposed that the $k$ th interlayer sliding belt consisted of $N$ elements. If the interlayer sliding belt is conform to the Markov stochastic process, then the failure probability of the $k$ th interlayer sliding belt is given by $p_{k}=p_{0} \prod_{j=2}^{N} p_{j \mid j-1}$ where $p_{k}$ is the failure probability of the k-interlayer sliding belt; $p_{j j-1}$ is conditional failure probability, which is defined as the failure probability of the $j$ th element when the $(j-1)$ th element is failed; and $p_{0}$ is the initial element failure probability. The markov process is usually used for calculating the failure probability of structure system and estimating its overall safety [11].

By analysis of the effects of probability distribution of the tensile strength of concrete $[12,13]$ considered that the normal distribution can reflect the probability distribution of tensile strength of concrete. Thus we assume that the tensile strengths of the first principal stress of elements obey the normal distribution. In other word, other distribution form can be translated into the enormal distribution easily, and normal distribution is extensively applied in the analysis of random variables.

\subsection{Mean Value and Variance of the Tensile Strength of Elements in Specified Interlayer Sliding Belts}

In the specified interlayer sliding belt, the failure of (i-1)element will decrease the tensile strength of $i$-element. Thus, the mean value of the tensile strength of i-element is given by

$$
\begin{aligned}
& E_{i}\left(f_{t}\right)=E_{0}\left(f_{t}\right)-\sum_{j=1}^{i-1} E_{j}\left(f_{t}\right) \\
& =E_{0}\left(f_{t}\right)-E_{0}\left(f_{t}\right) \times \sum_{j=1}^{i-1} p_{j \mid j-1}
\end{aligned}
$$

where $E_{0}\left(f_{\mathrm{t}}\right)$ is the mean value of tensile strength of the initial failure element in specified interlayer sliding belt and $E_{\mathrm{i}}\left(f_{\mathrm{t}}\right)$ is the mean value of tensile strength of i-element in the specified interlayer sliding belt.

And the variance of the tensile strength of i-element is given by

$D_{i}\left(f_{t}\right)=D_{0}\left(f_{t}\right)+D_{0}\left(f_{t}\right) \times \sum_{j=1}^{i-1} p_{j \mid j-1}^{2}$

where $D_{0}\left(f_{\mathrm{t}}\right)$ is the variance of tensile strength of the initial failure element in the specified interlayer sliding belt and $D_{\mathrm{i}}\left(f_{\mathrm{t}}\right)$ is the variance of tensile strength of i-element in the specified interlayer sliding belt.

\section{METHOD FOR CALCULATING CONDITIONAL FAILURE PROBABILITY}

In this study, we used the improved response surface method based on weighted regression $[14,15]$ to calculate conditional failure probability of elements in the specified interlayer sliding belt.
Generally, the failure mode of concrete under complex stress can be summarized as three types: tensile, compressive failure, and crushed failure under high hydrostatic pressures. But the early damage in concrete materials is mainly dominated by tensile failure. So the tensile failure of concrete is only considered in this paper, the tensile failure function of failure probability is given by

$g\left(f_{t}, \sigma_{1}\right)=f_{t}-\sigma_{1}$

where $f_{\mathrm{t}}$ is the tensile strength of elements and $\sigma_{1}$ is the first principal stress of elements.

Then, the tensile failure function of failure probability can be solved with the improved response surface method.

\section{ANALYSIS MODEL OF THE MEAN VALUE AND VARIANCE OF FIRST PRINCIPAL STRESS IN THE SPECIFIED INTERLAYER SLIDING BELT}

\subsection{The Pseudo Excitation Method Used for Calculating Auto-Spectral Density Function of Stress}

The autocorrelation $R_{x x}(\tau)$ of a random process $x(t)$ between the two different time is defined as

$$
\begin{aligned}
& R_{x x}(\tau)=E[x(t) x(t+\tau)] \\
& =\int_{-\infty}^{+\infty} \int_{-\infty}^{+\infty} x(t) x(t+\tau) d F(x, t ; x, t+\tau)
\end{aligned}
$$

where $E$ is the mean value operator, $t$ is time, $\tau$ is the time interval between the random process $x(t)$ and $x(t+\tau) . F$ is the probability density function.

Fourier transform pairs are consisted of auto-spectral density function $S_{x x}(f)$ and autocorrelation function $R_{x x}(\tau)$, it can be written as

$$
\begin{aligned}
& S_{x x}(f)=\int_{-\infty}^{\infty} R_{x x}(\tau) e^{-2 \pi i f \tau} d \tau \\
& R_{x x}(\tau)=\int_{-\infty}^{\infty} S_{x x}(f) e^{2 \pi i f \tau} d f
\end{aligned}
$$

where $f$ is the frequency of loads, and $e^{\mathrm{i}}$ is the operator of the complex variable function.

From above analysis, it can be seen that

$$
E_{x x}^{2}+D_{x x}^{2}=R_{x x}(0)=\int_{-\infty}^{\infty} S_{x x}(f) d f
$$

where $E_{x x}$ and $D^{2}{ }_{x x}$ denote the mean value and variance of $x(t)$. When $E_{x x}=0, D_{x x}^{2}$ can be determined by $S_{x x}(f)$.

Pseudo excitation method is the numerical methods for $S_{x x}(f)$, and the pseudo excitation $\tilde{x}(t)$ is given by

$\tilde{x}(t)=\sqrt{S_{x x}} e^{i \omega t}$

where $\omega$ is the angular velocity of load.

The relation between auto-spectral density function of response $S_{y y}(f)$ and the pseudo response $\tilde{y}(t)$ which is the response excited by the pseudo excitation $\tilde{x}(t)$, is given by

$$
\tilde{y}^{*} \tilde{y}=|\tilde{y}|^{2}=|H|^{2} S_{x x}=S_{y y}
$$


where $\tilde{y}^{*}$ is the conjugate function of $\tilde{y}$, and $H$ is transfer function between the pseudo excitation $\tilde{x}(t)$ and the pseudo response $\tilde{y}(t)$. When the response is the element stress, the auto-spectral density function of element stress is given by

$\boldsymbol{S}_{\sigma \sigma}=\{\tilde{\sigma}\}^{*} \cdot\{\tilde{\sigma}\}^{T}$

where $\tilde{\sigma}$ is the pseudo stress excited by the pseudo excitation.

\subsection{Calculation of Mean Value and Variance of First Principal Stress of Elements in Specified Interlayer Sliding Belt}

Firstly, the expected value of the first principal stress $E\left(\sigma_{1}\right)$ of elements in the specified interlayer sliding belt can be obtained by making static analysis. Then the variance of the first principal stress of element in the specified interlayer sliding belt is obtained as follows.

The vibration equation of the gravity dam system can be shown in the below form

$\boldsymbol{M} \ddot{\boldsymbol{V}}+\boldsymbol{C} \dot{\boldsymbol{V}}+\boldsymbol{K} \boldsymbol{V}=\boldsymbol{F}(t)$

where $\ddot{\boldsymbol{V}}, \dot{\boldsymbol{V}}$ and $\boldsymbol{V}$ are acceleration, velocity and displacement vectors; respectively $\boldsymbol{K}, \boldsymbol{C}$ and $\boldsymbol{M}$ are stiffness, damping and mass matrix; and $\boldsymbol{F}(t)$ is the seismic load.

The pseudo excitation $\tilde{F}(t)$ is written as

$\tilde{F}(t)=\sqrt{S_{f_{j}}(\omega)} e^{i \omega t}$

where $S_{f_{j}}(\omega)$ is the power spectrum of the seismic load $\boldsymbol{F}(t)$ and $j$ is $j$ th frequency.

The variance of stress $D(\sigma)$ can be obtained as

$D(\sigma)=\int_{-\infty}^{+\infty} S_{\sigma \sigma}(f) d f$

The relation between the first principal stress and other stresses can be expressed as

$$
\begin{aligned}
& \sigma_{1}=\frac{\left(\sigma_{x}+\sigma_{y}\right)+\sqrt{\left(\sigma_{x}-\sigma_{y}\right)^{2}+4 \tau_{x y}^{2}}}{2} \\
& \leq \max \left(\left|\sigma_{x}\right|+\left|\tau_{x y}\right|,\left|\sigma_{y}\right|+\left|\tau_{x y}\right|\right)
\end{aligned}
$$

where $\sigma_{1}$ is the first principal stress of elements. $\sigma_{x}$ and $\sigma_{y}$ are the element stresses in the $\mathrm{x}$ direction and $\mathrm{y}$ directions, respectively. $\tau_{x y}$ are the sheared stress.

Thus the upper limit of variance of the first principal stress of the $i$ th element in the specified interlayer sliding belt can be obtained as

$$
D\left(\sigma_{i 1}\right) \leq \max \left(D\left(\sigma_{i x}\right), D\left(\sigma_{i y}\right)\right)+D\left(\tau_{i x y}\right)
$$

In this paper, variance of the first principal stress of element in the specified interlayer sliding belt is used as the upper limit.

\section{NUMERICAL EXAMPLES}

We took a gravity dam, with the height of $160 \mathrm{~m}$, as an example. The water level of upstream of dam is $155 \mathrm{~m}$. The water level of downstream is $10 \mathrm{~m}$. The concrete strength of the gravity dam is $20 \mathrm{MPa}$. The finite element model of the gravity dam is divided into 2432 elements. The model of the dam and foundation consists of 8-node iso-parametric plane elements. The density of the dam $\rho=2450 \mathrm{~kg} / \mathrm{m}^{3}$, and Poisson ratio $v=0.18$. The damping ratio of dam $\zeta=0.05$. The density of rock foundation $\rho=2700 \mathrm{~kg} / \mathrm{m} 3$, and Poisson ratio $\nu=0.25$. Applied load includes gravity load and hydrostatic and uplift pressure and seismic load whose horizontal peak acceleration is $0.25 \mathrm{~g}$. The power spectrum density of seismic load is given by

$$
S_{f}\left(\omega_{k}\right)=\frac{2 \xi}{\pi \omega_{k}}\left[S_{a}^{T}\left(\omega_{k}\right)\right]^{2} \frac{1}{-2 \ln \left(-\frac{\pi}{\omega_{k} T_{d}} \ln p\right)}
$$

where $\omega_{k}$ is the angular velocity of of the $k$ th sample points, and $\omega_{k}=\triangle \omega k ; \triangle \omega$ is the interval of the angular velocity of number of sample points, and $\triangle \omega=2 \pi / T_{\mathrm{d}} ; S_{\mathrm{a}}^{\mathrm{T}}\left(\omega_{k}\right)$ is the target response spectrum; $\xi$ is the damping ratio; $p$ is the exceeding response spectrum probability, and $p \leq 0.15 ; T_{\mathrm{d}}$ is the duration of ground motion; $N$ is the number of trigonometric series, and $N=T_{\mathrm{d}} / \triangle t ; \triangle t$ are and time step. $k=1,2, \cdots, N$.

In this paper, the interlayer sliding belts were selected according to the most probably sliding surface in the concrete gravity dam, which are shown in the Figs. $(\mathbf{1}, \mathbf{2})$. It needs to be noticed that they are usually not the real interlayer sliding belts. The calculation process of the interlayer sliding belts is shown in Tables 1-3. Specifically, the specified interlayer sliding belts of gravity dam are shown in Fig. (1). The element numbers in the specified interlayer sliding belts of gravity dam are shown in Fig. (2). The calculation process of the failure probability of specified interlayer sliding belt on the downstream slope of the gravity dam is shown in Table $\mathbf{1}$. The calculation process of the failure probability of specified interlayer sliding belt on the upstream slope of the gravity dam is shown in Table $\mathbf{2}$. The calculation process of the failure probability of specified interlayer sliding belt on the foundation building surface of the gravity dam is shown in Table $\mathbf{3}$.

Eq. (16) is substituted into Eq. (11). Based on Eqs. (13) through (15), we can obtain the mean value and the mean variance of the first principal stress of element. With Eqs. (1) and (2), we can obtain the mean value and the mean variance of the tensile strength. And using the improved response surface method, we can obtain the conditional failure probability. And then we can obtain the results of Tables 1 and 2.

And the mean value, mean square deviation and conditional failure probability of first principle stress of elements in the specified interlayer sliding belts on the 


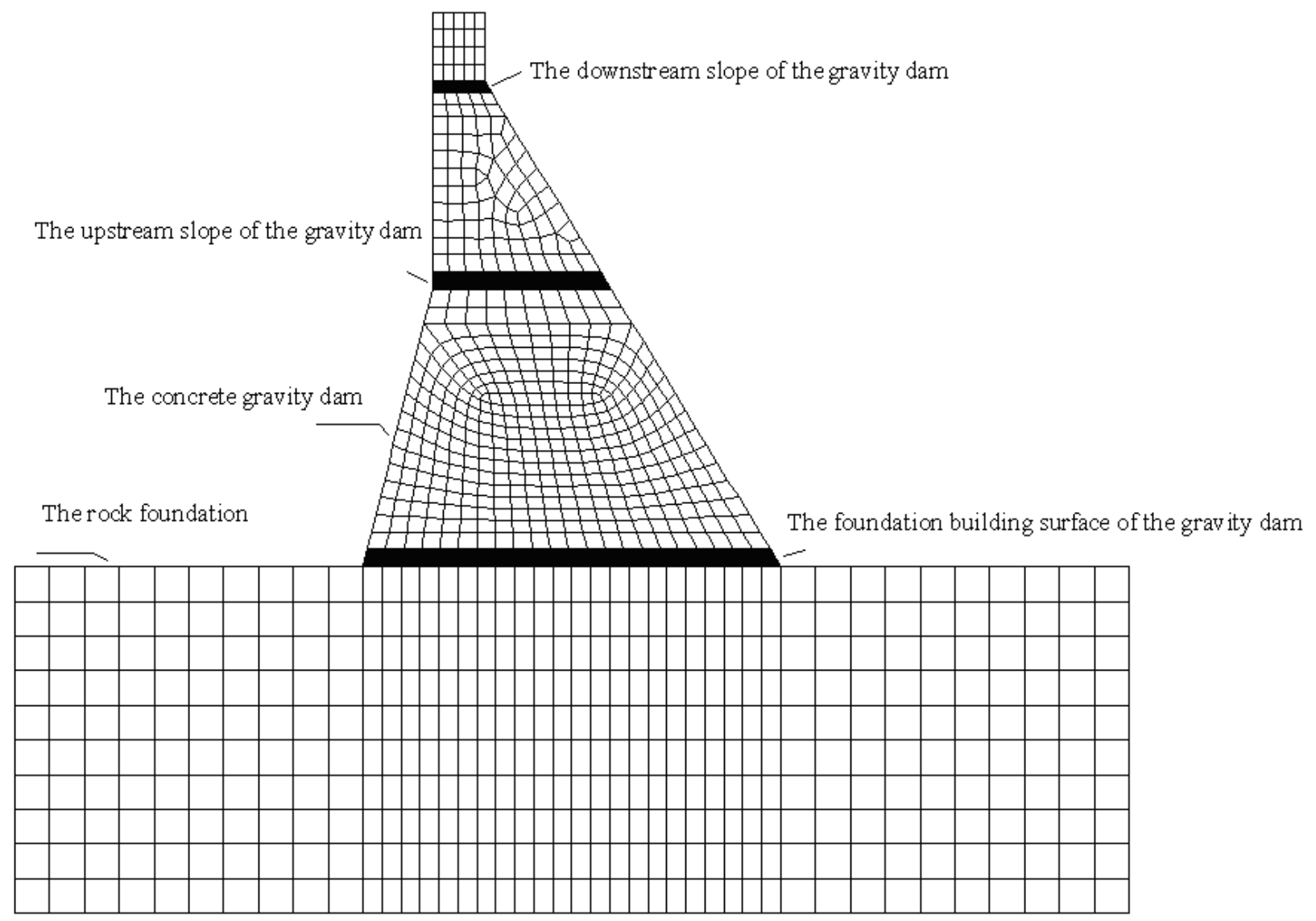

Fig. (1). The specified interlayer sliding belt of gravity dam.

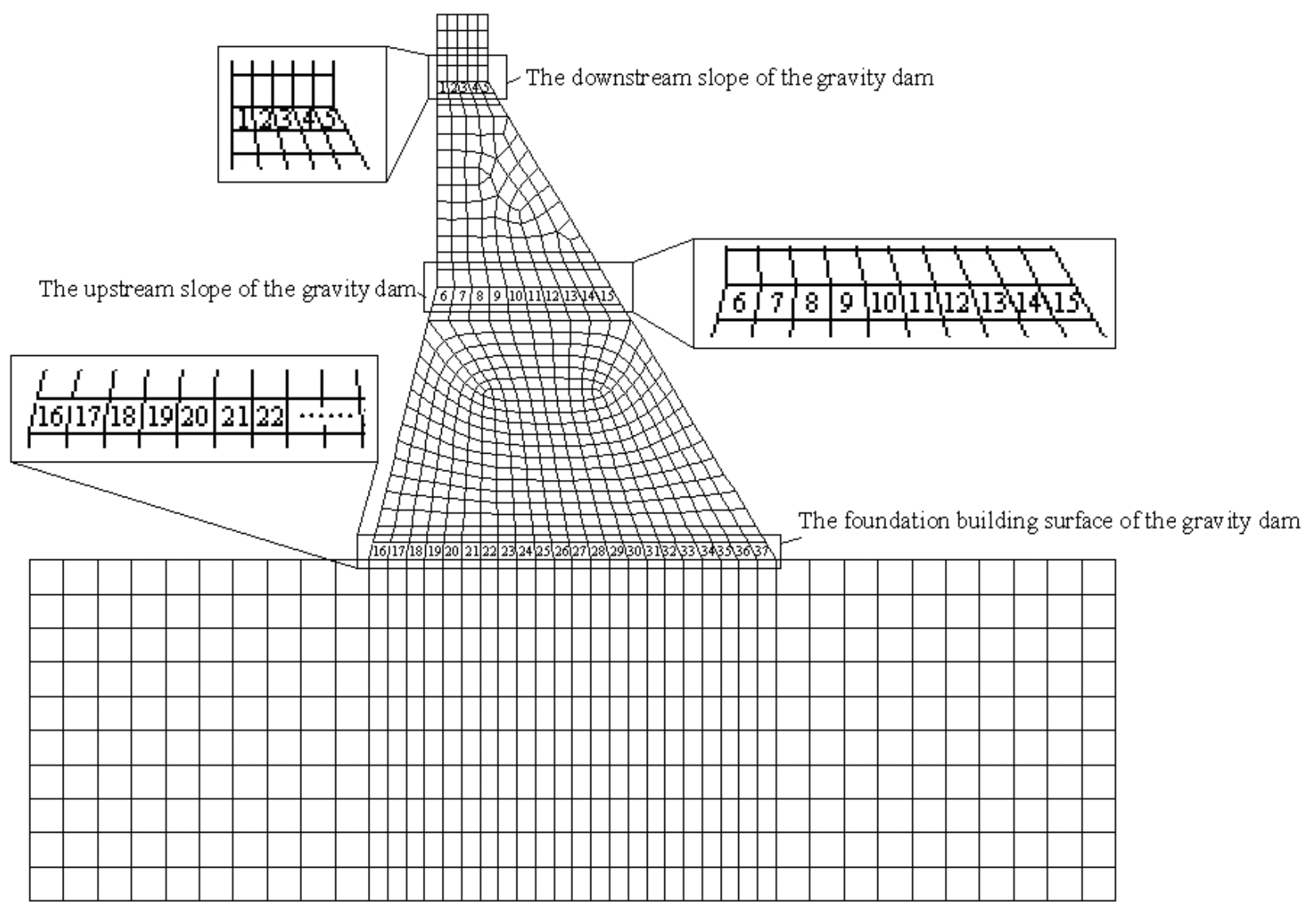

Fig. (2). The sketch for element numbers in the specified interlayer sliding belt of gravity dam. 
Table 1. The Specified Interlayer Sliding Belt on the Downstream Slope of the Gravity Dam

\begin{tabular}{|c|c|c|c|c|c|c|}
\hline Number of Elements & 1 & 2 & 3 & 4 & 5 & The Reliability Index in Specified Interlayer Sliding Belt \\
\hline $\mathrm{E}\left(\sigma_{1}\right)(\mathrm{MPa})$ & 0.067 & 0.059 & 0.044 & 0.025 & -0.004 & \multirow{3}{*}{2.45} \\
\hline$\sqrt{D\left(\sigma_{1}\right)}(\mathrm{MPa})$ & 1.856 & 1.424 & 1.317 & 1.646 & 2.673 & \\
\hline$p_{j \mid j-1}(\%)$ & 61.02 & 52.73 & 42.21 & 28.55 & 18.64 & \\
\hline
\end{tabular}

$E\left(\sigma_{1}\right)$ is the mean value of the first principal stress; $\sqrt{D\left(\sigma_{1}\right)}$ is the mean variance of the first principal stress.

Table 2. The Specified Interlayer Sliding Belt on the Upstream Slope of the Gravity Dam

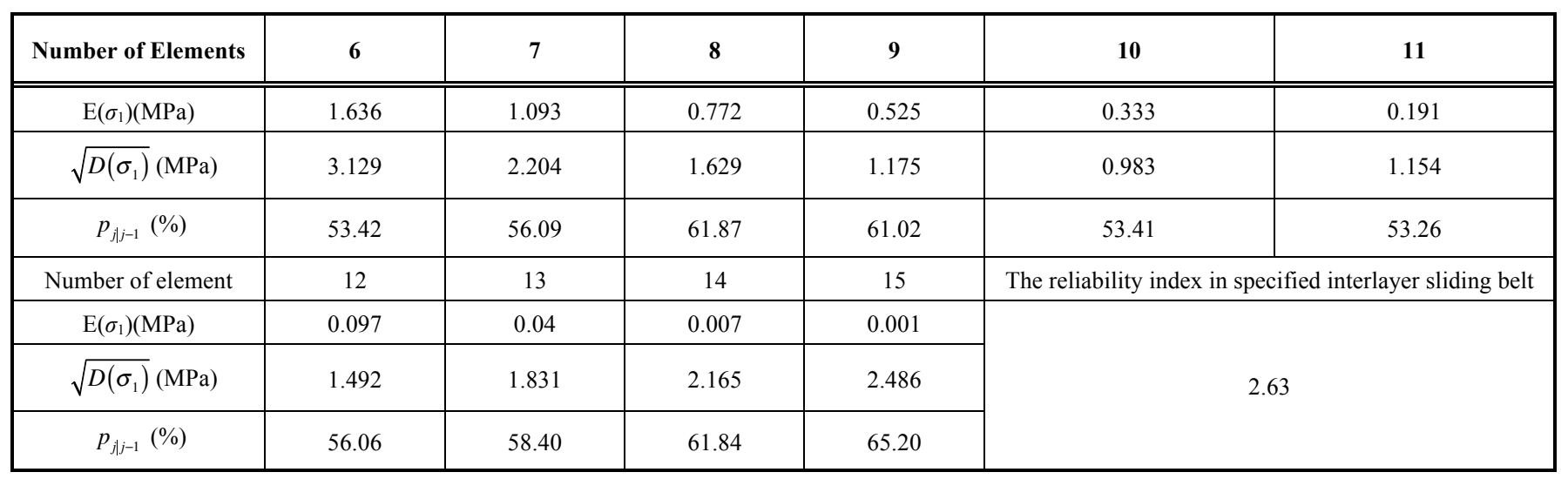

Table 3. The Specified Interlayer Sliding Belt on the Base Surface of the Gravity Dam

\begin{tabular}{|c|c|c|c|c|c|c|}
\hline $\begin{array}{l}\text { The number of elements in specified interlayer } \\
\text { sliding belt on the base surface of the gravity dam }\end{array}$ & 16 & 17 & 18 & 19 & 20 & 21 \\
\hline $\mathrm{E}\left(\sigma_{1}\right)(\mathrm{MPa})$ & 4.942 & 2.607 & 2.059 & 1.72 & 1.465 & 1.218 \\
\hline$p_{j \mid j-1}(\%)$ & 85.65 & 73.36 & 63.89 & 63.78 & 65.79 & 67.07 \\
\hline $\begin{array}{l}\text { The number of elements in specified interlayer } \\
\text { sliding belt on the base surface of the gravity dam }\end{array}$ & 22 & 23 & 24 & 25 & 26 & 27 \\
\hline$\sqrt{D\left(\sigma_{1}\right)}(\mathrm{MPa})$ & 1.226 & 1.115 & 1.019 & 0.956 & 0.96 & 1.018 \\
\hline$p_{j \mid j-1}(\%)$ & 69.99 & 68.76 & 70.93 & 73.81 & 76.09 & 78.78 \\
\hline $\begin{array}{l}\text { The number of elements in specified interlayer } \\
\text { sliding belt on the base surface of the gravity dam }\end{array}$ & 28 & 29 & 30 & 31 & 32 & 33 \\
\hline$p_{j \mid j-1}(\%)$ & 80.89 & 83.24 & 85.65 & 87.80 & 90.03 & 91.64 \\
\hline $\begin{array}{l}\text { The number of elements in specified interlayer } \\
\text { sliding belt on the base surface of the gravity dam }\end{array}$ & 34 & 35 & 36 & 37 & \multicolumn{2}{|c|}{ The reliability index in specified interlayer sliding belt } \\
\hline $\mathrm{E}\left(\sigma_{1}\right)(\mathrm{MPa})$ & -0.218 & -0.256 & -0.275 & -0.239 & \multirow{3}{*}{\multicolumn{2}{|c|}{2.53}} \\
\hline$\sqrt{D\left(\sigma_{1}\right)}(\mathrm{MPa})$ & 1.539 & 1.666 & 1.84 & 2.468 & & \\
\hline$p_{j \mid j-1}(\%)$ & 93.20 & 94.56 & 95.51 & 95.74 & & \\
\hline
\end{tabular}

downstream slope, upstream slope and the foundation building surface of the concrete gravity dam are shown in Tables 1-3.
From analysis of the calculation process of the interlayer sliding belts shown as Tables $\mathbf{1 - 3}$, the three interlayer sliding 
belts are considered as series structure for the concrete gravity dam system.

According to literature [16], concrete gravity dam can be regarded as parallel system. Based on the calculation principles for the failure probability of parallel structure, the failure probability of concrete gravity dam is

$P_{\text {system }}=1-\min (\varphi(2.45), \varphi(2.63), \varphi(2.53))=0.72 \%$

where $p_{\text {system }}$ is the failure probability of concrete gravity dam. $\varphi()$ is the function of the standard normal distribution.

Thus the failure probability of concrete gravity dam system is $0.72 \%$. The failure probability of concrete gravity dam is relatively high. The reason is that the proposed model considers the reduction of tensile strength of elements in the failure process. And the other reason is that the gravity dam is $160 \mathrm{~m}$ high, which is easily affected by earthquake load. The peak of earthquake load is used as $0.25 \mathrm{~g}$, which is relatively high.

From results, it can be also observed that the conditional failure probability in specified interlayer sliding belts at the head of the dam tends to descend. However, the tendency of conditional failure probability in the other specified interlayer sliding belts is complicated. And the interlayer sliding belt at head of the concrete gravity dam is the most dangerous. In addition, the tendencies of the mean value and mean square deviation of stress in different specified interlayer sliding belts are similar. The mean value of stress in different specified interlayer sliding belts tends to descend but the tendency of mean square deviation changes from decrease to increase. The range of the mean value and the mean square deviation of stress in specified interlayer sliding belts at the heel of the dam is the greatest of all.

\section{CONCLUSION}

An improved method is carried out for the analysis of failure probability of the interlayer sliding belt under seismic load. Firstly, based on the theory of Markov chain, the relation of failure probability between specified interlayer sliding belt and elements in this interlayer sliding belt is given. Then, the failure function is proposed according to the destructive character of concrete. Through above analysis, the pseudo excitation method is proposed in order to obtain the character of failure probability and the improved response surface method based on weighted regression is used to calculate the failure probability of elements in specified interlayer sliding belt. Finally, an algorithm is proposed to calculate the failure probability of the interlayer sliding belt. From analysis of the result of numerical examples, it can be obtained the conclusion that the conditional failure probability in specified interlayer sliding belt at the head of the concrete gravity dam tends to descend. However, the tendency of conditional failure probability in the other specified interlayer sliding belts is complicated. And the interlayer sliding belt at head of the concrete gravity dam is the most dangerous. In addition, the tendencies of the mean value and mean square deviation of first principle stress in different specified interlayer sliding belts are similar. The mean value of first principle stress in different specified interlayer sliding belts tends to descend but the tendency of mean square deviation of first principle stress in different specified interlayer sliding belts changes from decrease to increase. The range of the mean value and the mean square deviation of first principle stress in specified interlayer sliding belt on the foundation building surface at the heel of the concrete gravity dam is the greatest of those in different specified interlayer sliding belts.

\section{CONFLICT OF INTEREST}

The authors confirm that this article content has no conflict of interest.

\section{ACKNOWLEDGEMENTS}

This study was supported by Project supported by the National Natural Science Foundation of China (Grant No. 51109029, 51178081, 51138001, 51009020), the State Key Development Program for Basic Research of China (No.2013CB035905).

\section{REFERENCES}

[1] Y.L. Xu, W.S. Zhang, J.M. Ko, and J.H. Lin, "Pseudo-excitation method for vibration analysis of wind-excited structures," Journal of Wind Engineering and Industrial Aerodynamics, vol. 83(1-3), pp. 443-454, 1999.

[2] S. D. Xue, Z. Cao, and X. S. Wang, "Random vibration study of structures under multi- component seismic excitations," Advances in Structural Engineering, vol. 5(3), pp. 185-192, 2002.

[3] Q.S. Li, Y.H. Zhang, J.R. Wu, and J.H. Lin, "Seismic random vibration analysis of tall buildings," Engineering Structures, vol. 26(12), pp. 1767-1778, 2004.

[4] J.H. Lin, Y.H. Zhang, Q.S. Li, and F.W. Williams, "Seismic spatial effects for long-span bridges using the pseudo excitation method," Engineering Structures, vol. 26 (9), pp. 1207-1216, 2004.

[5] M.F. Wang, "Pseudo-excitation method for stationary random responses of non- proportionally damped MDOF systems," Chinese Journal of Computational Mechanics, vol. 25(1), pp. 94-99, 2008.

[6] W. T. XU, J. H. Lin, Y. H. Zhang, D. Kennedy, and F. W. Williams, "Pseudo-excitation method based sensitivity analysis and optimization for vehicle ride comfort," Engineering Optimization, vol. 41(7), pp. 699-711, 2009.

[7] J. Li, Y.Y. Qin, and Q. Zhao, "Pseudo-excitation method and its application to vehicle ride comfort," SAE World Congress \& Exhibition, vol. 1(1), pp. 12-21, 2009.

[8] J. H. Lin, Y. H. Zhang, and Y. Zhao, "Pseudo excitation method and its application in seismic design and disaster mitigation of long-span structures," Earth and Space: Engineering, Science, Construction, and Operations in Challenging Environments, vol.10(1), pp. 2941-2948, 2010.

[9] W. Sun, W.T. Xu, J.H. Lin, D. Kennedy, and F.W. Williams, "Ride-comfort-oriented suspension optimization using the pseudoexcitation method," Journal of Automobile Engineering, vol. 224(11), pp. 1357-1367, 2010

[10] B.S Liu, G. Z. Zhao, and R.Y. Li, PEM based acoustic radiation analysis from random excited structure," Chinese Journal of Solid Mechanics, vol. 32(6), pp. 581-587, 2011

[11] Z.Q. Zhang and C.G. Wu, "Reliability analysis methods on the safety related systems," Journal of Safety and Environment, vol. 2, no. 2, pp. 47-50, 2002 .

[12] J.Y. Chen, and W.F. Bai, "Statistical damage model of concrete under uniaxial tension considering dynamic strain-rate effect," Chinese Journal of Rock Mechanics and Engineering, vol. 26(8), pp. 1603-1611, 2007. 
[13] M.D. Feng, J.B. Li, G. Lin, and Z.G. Liu, "Influence of stochastic mechanical parameters on meso-damage evolution of concrete," Journal of Architecture and Civil Engineering, vol. 27 (4), pp. 1-6, 2010.

[14] J.Y. Chen, Q. Xu, J. Li, and S.L. Fan, "Improved response surface method for anti-slide reliability analysis of gravity dam based on weighted regression", Journal of Zhejiang University -SCIENCE A (Applied Physics \& Engineering), vol. 11(6), pp.432-439, 2010.

[15] Q. Xu, J. Li and J.Y. Chen, "Probability analysis for the damage of gravity dam”, Journal of Engineering, vol. 3(4), pp. 312-321, 2011.

G.F. Zhao, J.X. Gong and S.C. Zhao, "Developments of reliability research for civil engineering structure in China," Journal of Dalian University of Technology, vol. 40(3), pp. 253-258.

(C) Xu et al.; Licensee Bentham Open.

This is an open access article licensed under the terms of the Creative Commons Attribution Non-Commercial License (http://creativecommons.org/licenses/ by-nc/3.0/) which permits unrestricted, non-commercial use, distribution and reproduction in any medium, provided the work is properly cited. 